\title{
Regulador cuadrático lineal y control predictivo aplicados en un sistema de cuatro tanques: una comparación de rendimiento
}

\section{(Linear Quadratic Regulator and Model Predictive Control Applied to a Four-Tank System: A Performance Comparison)}

\author{
William Chamorro ${ }^{1}$, Pablo Velarde Rueda ${ }^{1}$
}

\begin{abstract}
Resumen:
Este documento presenta una comparación entre dos controladores lineales conocidos como PI-LOR y MPC con restricciones suaves aplicados a un proceso de cuatro tanques acoplados, cuyas características principales son las no-linealidades, múltiples entradas y salidas y una dinámica lenta. El modelo es linealizado alrededor de un punto de funcionamiento mediante mínimos cuadrados. Además, se presenta una formulación matemática general para ambos controladores que puede ser fácilmente extendida a cualquier control de procesos. Todo el procedimiento es descrito en detalle, así como los resultados de la simulación para ambos controladores. Para lograr una comparación real del rendimiento, los datos experimentales son obtenidos en las mismas condiciones y se analizaron todos los parámetros de respuesta.
\end{abstract}

Palabras clave: MPC; LQR; control óptimo; mínimos cuadrados; control de procesos; sistema de cuatro tanques.

\begin{abstract}
:
This paper shows a comparison between two linear controllers, a PI-LQR and a SoftConstrained MPC applied to a Four-Tank process, which main characteristics are the nonlinearities, multiple inputs and outputs coupled together and slow behavior. The model was linearized around an operating point by least square method. Also, a general mathematic formulation for both controllers is presented that can be extended to any process control. All the procedure was described in detail as well as the simulation results for both controllers. To achieve a real performance comparison the experimental data were obtained under the same conditions and all the response parameters were analyzed.
\end{abstract}

Keywords: MPC; LQR; optimal control; least square method, process control; 4-tank system.

\section{Introducción}

Las aplicaciones industriales generalmente requieren interacciones entre reactivos, donde un nivel de líquido debe ser constante para alcanzar una reacción química con éxito. Estos procesos industriales se desarrollan en sistemas que involucran tanques acoplados entre sí. Los sistemas de dos tanques son ampliamente utilizados en procesos de purificación de agua, procesamiento de alimentos, tratamientos de desechos nucleares, entre otros. Estos ejemplos incluyen requisitos de rendimiento agresivos que pueden no ser alcanzables con métodos de control en lazo abierto (Vikram, et al., 2005). Los sistemas acoplados con 3 o 4 tanques se han convertido en una herramienta útil en el tratamiento de bio-contaminantes (Guillod, et al., 2003) o aguas residuales con alto contenido orgánico (Mavrias, et al., 2003). Es por eso que, desarrollar estrategias de

\footnotetext{
1 Universidad Tecnológica Equinoccial, Quito, Ecuador ( \{william.chamorro, pablo.velarde\} @ute.edu.ec).
} 
control para superar las irregularidades o limitaciones en los procesos industriales son casos de estudio llamativos dentro de la ingeniería de control.

En el campo de control, se ha prestado especial atención a los sistemas de cuatro tanques introducidos por (Johansson, 2000). Debido a su versatilidad para aplicaciones educativas e industriales. El sistema ofrece una dinámica no lineal y variables físicas acopladas que hacen atractivo llevar a cabo varias estrategias de control como se muestra en (Cheng, et al., 2016); (Askari, et al., 2016). Dentro de esta variedad, dos técnicas de control han sido ampliamente utilizadas por su robustez: control predictivo basado en el modelo (Model Predictive Control) y regulador cuadrático lineal (Linear Quadratic Regulator) conocidos como MPC y LQR por sus siglas en inglés, respectivamente. Existen técnicas de optimización basadas en algoritmos genéticos como en el trabajo realizado en (Najarro, et al., 2017), que pueden ser tratados a futuro.

MPC es una estrategia de control utilizada en varios campos industriales debido a su adaptabilidad. El controlador MPC puede considerar retardos en las entradas y salidas, no linealidades, incertidumbres, naturaleza estocástica, por nombrar algunos (Grosso, et al., 2016); (Camacho \& Bordons, 2004); (Velarde, et al., 2017). La idea detrás de este esquema de control es minimizar una función objetivo mediante el cálculo de una secuencia de acciones de control a lo largo de un horizonte de predicción; solo el primer componente de esta secuencia de control se implementa en el paso de tiempo actual; las acciones de control restantes se descartan. El problema de optimización se repite a cada instante del tiempo. Este esquema permite obtener una señal de control basada en estados pasados así como en estados futuros fundamentado en un modelo del sistema dinámico.

LQR es un método sustentado en la ponderación de las variables de estado y las entradas de control. Además, esta técnica ofrece un menor consumo de recursos computacionales en su implementación. Las respuestas producidas son más rápidas con una acción de control óptima, especialmente en los sistemas de tanques acoplados (Engules, et al., 2015). En este sentido, la carga computacional es reducida notablemente en su implementación.

El presente artículo propone una comparación adecuada entre estas dos estrategias de control ampliamente implementadas. La comparación busca mostrar sus ventajas y desventajas, así como presentar sus diseños e implementación en un caso de estudio real. Con este fin, el sistema de cuatro tanques acoplados se modela al tener en cuenta sus no linealidades. Sin embargo, para probar los controladores diseñados es necesario considerar un modelo lineal alrededor de un punto de operación adecuado, que se detalla en este trabajo. El sistema de cuatro tanques acoplados puede ser muy vulnerable al presentar un error de posición en estado estacionario; este inconveniente se puede superar mediante la introducción de restricciones suaves (soft-constraints) y una acción integral en la formulación tradicional para los controladores MPC y LQR, respectivamente; dando como resultado un MPC con restricciones suaves (SC-MPC) y la acción integral en el LQR (PI-LQR). Estas dos formulaciones y el proceso de linealización, son descritas en detalle y generalizadas para cualquier sistema.

El resto de este documento está organizado de la siguiente manera. La Sección 2 presenta la descripción no lineal del sistema de cuatro tanques, el modelo matemático, los parámetros de trabajo y su linealización referida a un punto de trabajo obtenido a partir de una curva de reacción. En la Sección 3, las dos técnicas de control PI-LQR y SC-MPC son formuladas bajo una manera general y ser aplicadas en el sistema descrito. Los resultados de la comparación que señalan ventajas y desventajas entre estas dos técnicas, se proporcionan en la Sección 4. Finalmente, las conclusiones son especificadas en la sección 5 . El presente trabajo es una versión extendida y en español del artículo (Chamorro, et al., 2017). 


\section{Descripción del sistema de cuatro tanques acoplados}

La planta de cuatro tanques es un sistema educativo diseñado para probar técnicas de control mediante instrumentación de tipo industrial (Alvarado, et al., 2011). El sistema consiste en una modificación de los cuatro tanques de agua interconectados presentados por (Johansson, 2000) para realizar el seguimiento de referencias mediante SC-MPC y PI-LQR. La Figura 1 muestra el diagrama de la planta, que se compone de cuatro tanques: dos tanques superiores ( 3 y 4 ), que se descargan en dos inferiores ( 1 y 2 ). Cada tanque se llena con el flujo de un tanque de almacenamiento situado en la parte inferior de la planta mediante dos bombas $\left(q_{A} \mathrm{y} q_{B}\right)$. Los flujos de entrada están regulados por válvulas de tres vías. El sistema se modela como se muestra a continuación:

$$
\begin{gathered}
\frac{d h_{1}}{d t}=-\frac{a_{1}}{S_{1}} \sqrt{2 g h_{1}}+\frac{a_{3}}{S_{3}} \sqrt{2 g h_{3}}+\frac{\gamma_{a}}{S_{1}} q_{A} \\
\frac{d h_{1}}{d t}=-\frac{a_{2}}{S_{2}} \sqrt{2 g h_{2}}+\frac{a_{4}}{S_{4}} \sqrt{2 g h_{4}}+\frac{\gamma_{b}}{S_{2}} q_{B} \\
\frac{d h_{1}}{d t}=-\frac{a_{3}}{A_{3}} \sqrt{2 g h_{3}}+\frac{\left(1-\gamma_{b}\right)}{S_{3}} q_{A} \\
\frac{d h_{1}}{d t}=-\frac{a_{4}}{s_{4}} \sqrt{2 g h_{4}}+\frac{\left(1-\gamma_{a}\right)}{S_{4}} q_{B}
\end{gathered}
$$

Donde $h_{\{1,2,3,4\}}$ es el nivel de flujo de cada uno, S es la sección transversal, $a_{\{1,2.3 .4\}}$ es la constante de descarga y $\gamma_{\{A, B\}}$ es la relación de la válvula de tres caminos.

\section{Sistema de 4 tanques acopados}

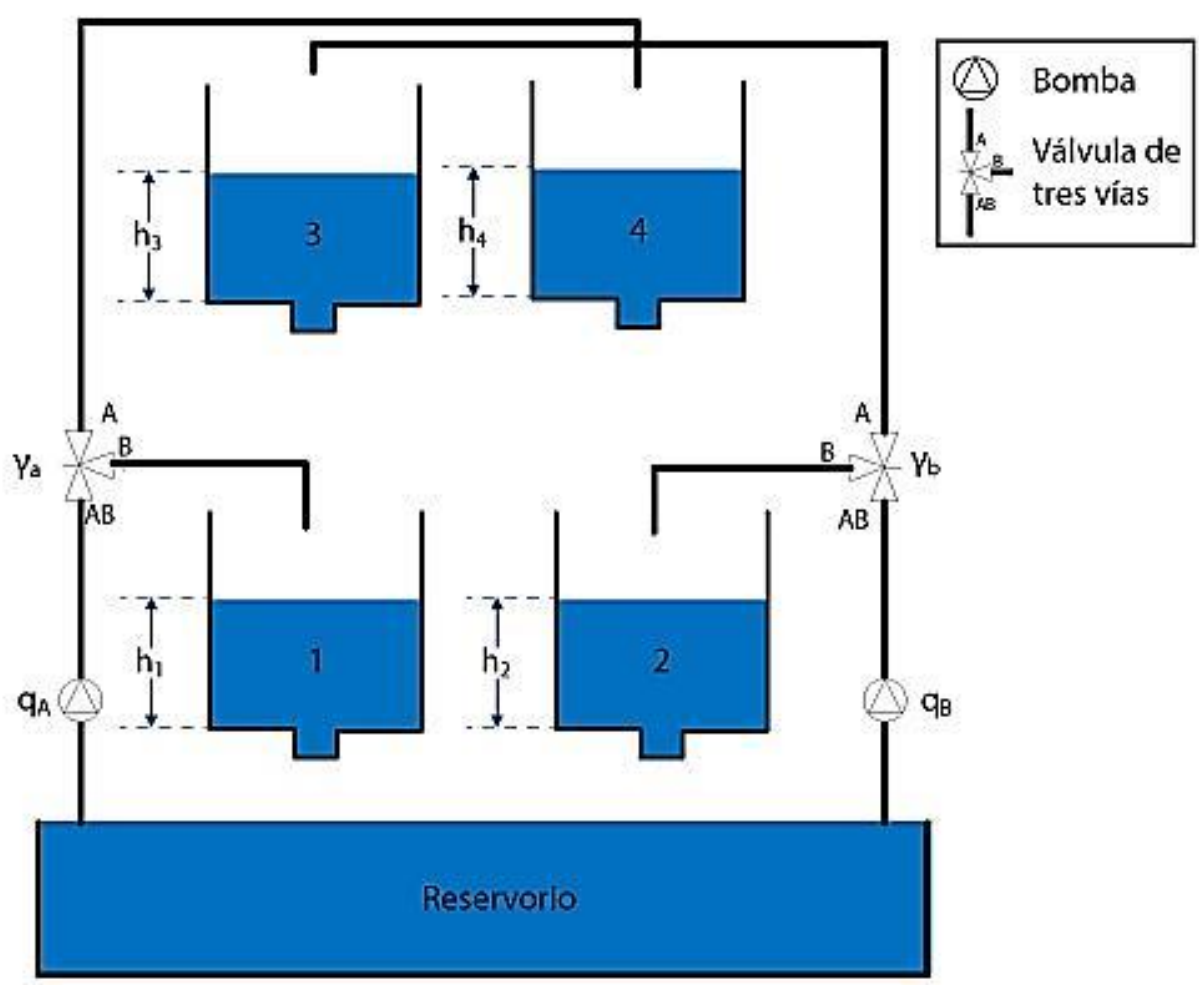

Figura 1. Diagrama de la planta de 4 tanques acoplados

La Tabla 1 muestra los parámetros empleados en el proceso de simulación 
Tabla 1. Parámetros del sistema

\begin{tabular}{|c|c|c|c|}
\hline Parámetro & Valor & Parámetro & Valor \\
\hline$h_{1}^{0}$ & $0.65 \mathrm{~m}$ & $a_{1}$ & $1.31 \times 10^{-4} \mathrm{~m}^{2}$ \\
\hline$h_{2}^{0}$ & $0.65 \mathrm{~m}$ & $a_{2}$ & $1.507 \times 10^{-4} \mathrm{~m}^{2}$ \\
\hline$h_{3}^{0}$ & $0.65 \mathrm{~m}$ & $a_{3}$ & $9.627 \times 10^{-4} \mathrm{~m}^{2}$ \\
\hline$h_{4}^{0}$ & $0.65 \mathrm{~m}$ & $a_{4}$ & $8.31 \times 10^{-4} \mathrm{~m}^{2}$ \\
\hline$q_{A}^{0}$ & $1.63 \mathrm{~m}^{3} / \mathrm{h}$ & $\gamma_{a}$ & 0.30 \\
\hline$q_{B}^{0}$ & $2 \mathrm{~m}^{3} / \mathrm{h}$ & $\gamma_{b}$ & 0.40 \\
\hline$A_{\{1,2.3 .4\}}$ & $0.06 \mathrm{~m}^{2}$ & & \\
\hline
\end{tabular}

Para asegurar el correcto funcionamiento de la planta y su equipo, el sistema está sujeto a restricciones en cada instante de tiempo $k \in \mathrm{Z}_{+}$, es decir:

$$
\begin{gathered}
0.2 m \leq h_{1}[k] \leq 2 m, \forall_{i} \in 1,2,3,4 \\
0 m^{3} / h \leq q_{A}[k] \leq 3.26 m^{3} / h \\
0 \frac{m^{3}}{h} \leq q_{B}[k] \leq 4 \frac{m^{3}}{h}
\end{gathered}
$$

Estas restricciones se pueden expresar como restricciones en los estados, en las entradas o como restricciones politópicas.

$$
\begin{aligned}
& x[k] \in X \\
& x[k] \in U
\end{aligned}
$$

La función de coste para este sistema está dado por:

$$
J(x[k], u[k])=\sum\left(x[k]-x_{r e f}\right)^{T} Q\left(x[k]-x_{r e f}\right)+u^{T}[k] R u[k]
$$

Donde, $x_{r e f}$ es el nivel de referencia dado para los estados, $Q$ y $R$ son los pesos para los estados y las entradas de control, respectivamente.

\subsection{Modelo del sistema lineal}

El nivel en cada uno de los cuatro tanques se estabiliza en un valor de altura que depende de los dos flujos en las entradas del sistema. La Figura 2 representa la curva de reacción; esta curva se obtiene al proporcionar varias entradas que van desde 0 hasta los valores de flujos máximos dados por las restricciones propias del sistema. Además, permite identificar el punto de trabajo, denominado también punto de operación, alrededor del cual el sistema será linealizado y hasta qué punto puede extenderse la linealización para seguir con un modelo lineal válido.

Como se puede observar en la Figura 2, el punto de operación $h^{0}=$ $[0.65,0.65,0.65,0.65] \mathrm{m}$; ubicado en la mitad de la curva, permite; desplazamientos lineales a lo largo de una sección recta.

Un método para la identificación de sistemas no lineales se muestra en (Sugeno \& Takagi, 1985), mediante la optimización un índice de rendimiento. Esta técnica consiste en generar un conjunto compuesto por $\eta$ muestras aleatorias alrededor del punto de operación como datos de entrada y $\eta$ salidas correspondientes a la respuesta a las entradas dadas. Ambos datos de entrada y salida se calculan mediante el modelo no lineal. Estos valores son la base para el algoritmo de linealización. Por lo tanto, cada muestra puede ser expresada según las ecuaciones 8 a la 15. 


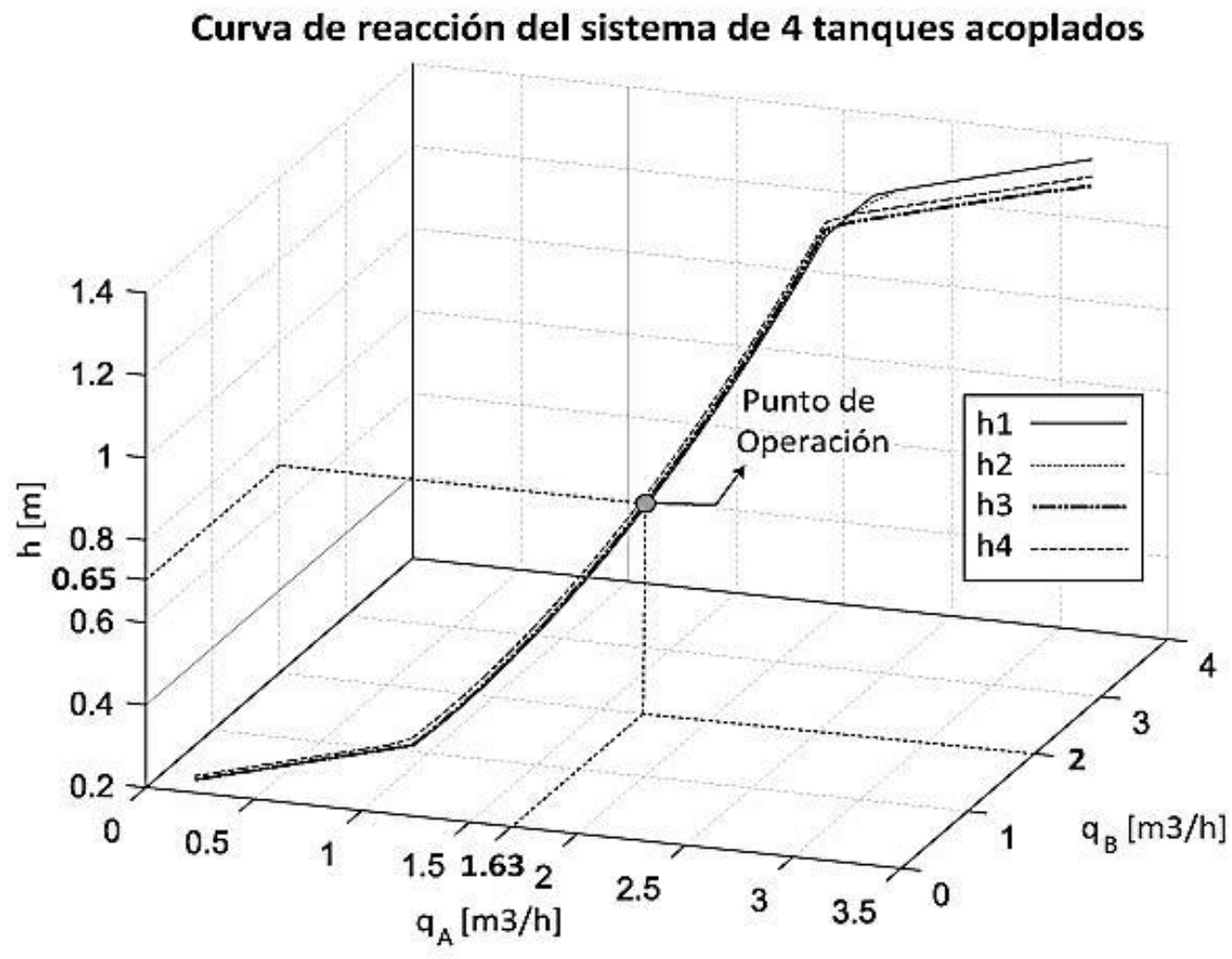

Figura 2. Curva de reacción del sistema

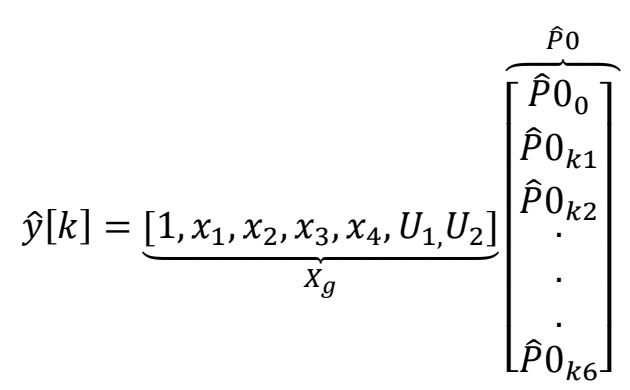

Donde, $\widehat{P} 0$ son las constantes de linealización, obtenidas al alcanzar el menor error en la estimación de salida. Este error está dado por:

$$
e(k)=y([k])-X_{g} * \hat{P} 0
$$

Al reducir un índice de rendimiento, el error cuadrado de la matriz de parámetros también es minimizada (Jimenez, et al., 2013). Este índice es:

$$
\begin{gathered}
\Phi=\|e(k)\|^{2} \\
\Phi=\frac{1}{2} * e^{T} * e \\
\frac{\partial \Phi}{\partial \hat{P} 0}=\frac{\partial\left(y[k]-X_{g} * \hat{P} 0\right)^{T} *\left(y[k]-X_{g} * \hat{P} 0\right)}{2 \partial \hat{P} 0}
\end{gathered}
$$

Se logra una mayor precisión con un mayor número de muestras durante el proceso de estimación, además, $X_{g}$ es una matriz de rango completo por lo tanto la ecuación (12) está expresada de la siguiente manera:

$$
\hat{P} 0=X_{g}{ }^{T} * X_{g}{ }^{-1} * X_{g}{ }^{T} * y(k) ; \in \mathbb{R}^{\left(n_{x}+n_{u}\right) \times\left(n_{x}\right)}
$$


Las matrices $A$ y $B$ están contenidas dentro de $\hat{P} 0^{T}$. El modelo lineal en tiempo discreto del sistema de cuatro tanques alrededor de los puntos de trabajo $q_{A}^{0}, q_{B}^{0}$ y $h_{i}^{0}$ descritos en la Tabla 2, se expresa como:

$$
x[k+1]=A x[k]+B u[k]
$$

Donde $x[k]=h_{i}[k]-h_{i}^{0}$, para $i=\{1,2,3,4\}$ y $u_{1}[k]$ y $u_{2}[k]$ corresponde a $\left[q_{A}[k]-\right.$ $\left.q_{A}^{0} ; q_{B}[k]-q_{B}^{0}\right]$. Además, A y $\mathrm{B}$ son matrices invariantes en el tiempo, que pueden obtenerse de la solución (13) de la siguiente manera:

$$
\hat{P} 0^{T}=\left[A \in \mathbb{R}^{n_{x} \times n_{x}} \| B \in \mathbb{R}^{n_{z} \times n_{u}}\right.
$$

En particular, para este modelo lineal, $n_{x}=4$ y $n_{u}=2$. En este punto las matrices $A$ y $B$ para un tiempo de muestreo de $T=5 \mathrm{~s}$, son definidas como:

$$
A=\left[\begin{array}{cccc}
0.9375 & 0 & 0.0442 & 0 \\
0 & 0.9280 & 0 & 0.0421 \\
0 & 0.9280 & 0.9558 & 0 \\
0 & 0 & 0 & 0.9579
\end{array}\right], B=\left[\begin{array}{cc}
0.0139 & 0 \\
0 & 0.0185 \\
0 & 0.0278 \\
0.0324 & 0
\end{array}\right]
$$

\section{Diseño de controladores}

El sistema de cuatro tanques consta de múltiples entradas y múltiples salidas (MIMO) con error en estado estable diferente de cero, la comparación del rendimiento se lleva a cabo entre los dos controladores propuestos que buscan reducir este error. En esta sección se formula el problema de control para el sistema mencionado anteriormente basado en un PI-LQR y SC-MPC.

\subsection{Regulador cuadrático lineal con acción integral}

El sistema lineal, definido por (14) puede ser llevado desde un estado inicial $x[0]$ a otro $x[k]$ por medio de una acción de control bajo una formulación en lazo cerrado dada por:

$$
u[k]=-K * x[k]
$$

Donde $K \in \mathbb{R}^{\text {nu } \times \mathrm{nx}}$ es una matriz de ganancias. Para determinar $\mathrm{K}$, el siguiente criterio (función objetivo) debe ser minimizado (Herrera, et al., 2015),

$$
J=\sum\left[x^{T}[k] Q x[k]+u^{T}[k] R u[k]\right]
$$

La solución a este problema es encontrar la matriz $\mathrm{P}$, definido-positivo que resuelve la ecuación de Ricatti:

$$
P * B * R^{-1} * B^{T} * P-P * A * A^{T} * P-Q=0
$$

La ganancia de la matriz K es calculada de la siguiente manera:

$$
K=R^{-1} * B^{T} * P
$$

Para garantizar un error de posición en estado estable igual a cero, se debe agregar una acción integral al controlador LQR. Aquí, las referencias dadas al sistema se denotan como $x_{\text {ref }}$. Se propone agregar nuevos estados $\omega \in \mathbb{R}^{\mathrm{nx}}$ para lograr una acción integral asociada con el error entre cada instante de tiempo $[k]$ y el consecutivo $[k+1]$, para más detalle véase (Jimenez, et al., 2013).

$$
\begin{gathered}
w[k+1]=w[k]+x_{r e f}-x[k+1] \\
w[k+1]=w[k]+x_{r e f}-(A-B * k) * x[k]
\end{gathered}
$$

La ecuación (21) representa un sistema extendido cuya formulación se puede escribir como:

$$
\left[\begin{array}{c}
w[k+1] \\
x[k+1]
\end{array}\right]=\left[\begin{array}{cc}
I & -C \\
0 & A
\end{array}\right]\left[\begin{array}{c}
w[k] \\
x[k]
\end{array}\right]+\left[\begin{array}{l}
0 \\
B
\end{array}\right] u[k]+\left[\begin{array}{l}
I \\
0
\end{array}\right] x_{\text {ref }}
$$


El sistema dado por (22) es reescrito como:

$$
\begin{gathered}
x_{a}[k+1]=A_{a} x_{a}[k]+B_{a} u[k]+B_{y} x_{r}[k] \\
y[k]=C_{a} x_{a}[k]
\end{gathered}
$$

Donde, $A_{a} \in \mathbb{R}^{\left(2 n_{x}\right) \times\left(2 n_{x}\right)}, B_{a} \in \mathbb{R}^{\left(2 n_{x}\right) \times\left(n_{u}\right)}, B_{y} \in \mathbb{R}^{\left(2 n_{x}\right) \times\left(n_{x}\right)}$, y $C_{a} \in \mathbb{R}^{\left(n_{x}\right) \times\left(2 n_{x}\right)}$, $n_{x}=4$ y $n_{u=}=2$. El índice de rendimiento que considera el sistema extendido se convierte

$$
J=\sum_{k=0}^{N}\left[\left(x_{r e f}-x[k]\right)^{T} *\left[\begin{array}{cc}
Q_{w} & 0 \\
0 & Q_{x}
\end{array}\right] *\left(x_{r}-x[k]\right)+u^{T}[k] * R * u[k]\right]
$$

Al priorizar el valor de los estados frente a las acciones de control, los valores $R$ y $Q$ seleccionados para el algoritmo del controlador LQR discreto son:

$$
R=\left[\begin{array}{cc}
0.1 & 0 \\
0 & 0.1
\end{array}\right], Q=\left[\begin{array}{cc}
Q_{w} & 0 \\
0 & Q_{x}
\end{array}\right] \text { donde, } Q_{w}=I_{n_{x} \times n_{x}}, Q_{x}=I_{n_{x} \times n_{x}}
$$

\subsection{Control predictivo con restricciones suaves}

MPC es una estrategia de control basada en el uso del modelo lineal dinámico del sistema para calcular la evolución de los estados a lo largo de un horizonte de predicción $\left(N_{p}\right)$. La secuencia de acciones de control futuras $\left(\left\{u[k], \ldots, u\left[k+N_{p}-\right.\right.\right.$ 1]\}) se calcula minimizando la función de coste dada por (5) a lo largo de $N_{p}$. Solo el primer elemento de la secuencia $(u[k])$ se aplica al proceso en el instante de tiempo actual $k$; los elementos restantes son descartados. El problema de optimización se repite en el siguiente instante de tiempo $(k+1)$ para calcular las acciones correspondientes con la información más reciente. manera:

El problema de optimización resuelto en cada instante $k$ se formula de la siguiente

Sujeto a:

$$
\{u[k], \ldots, u[k+N-1]\} \sum_{i=k}^{\min } J(x[i+1], u[k]
$$

$$
\begin{gathered}
x(i+1)=A x(i)+B u(i)+w(i), \forall i \in \mathbb{Z}_{k}^{k+n_{p}-1} \\
x[i+1] \in X, \forall i \in \mathbb{Z}_{k}^{k+n_{p}-1} \\
x[i] \in U, \forall i \in \mathbb{Z}_{k}^{k+n_{p}-1}
\end{gathered}
$$

Donde $\mathbb{Z}_{0}^{N-1}$ es el conjunto de números enteros de 0 a $N_{p}-1$.

Una alternativa para conseguir el seguimiento de la referencia es incorporar restricciones suaves en el problema de optimización. La idea principal detrás de la introducción de restricciones suaves es considerar un sistema extendido que replica los estados $\left(x[k] \in \mathbb{R}^{n x}\right)$ y las entradas de control $\left(u[k] \in \mathbb{R}^{n u}\right)$ del sistema físico y adicionar acciones de control "virtuales" en las entradas del sistema $\left(\tilde{u}[k] \in \mathbb{R}^{n x}\right)$, una por cada estado, que actúan libremente cuando los estados replicados $\left(\tilde{x}[k] \in \mathbb{R}^{x}\right)$; altamente penalizados cuando los estados se encuentran lejos de las referencias. En este sentido, la función objetivo se puede escribir como:

$$
J(\hat{x}[k+1], \hat{u}[k])=\left(\hat{x}[k+1]-\hat{x}_{r e f}\right)^{T} \hat{Q}\left(\hat{x}[k+1]-\hat{x}_{r e f}\right)+\hat{x}^{T} \hat{R} \hat{u},
$$

Donde $\tilde{x}[k+1]$ y $\tilde{u}[k]$ representan los estados extendidos y la entrada de control, respectivamente. Por lo tanto, el sistema aumentado se define como:

$$
\begin{gathered}
\hat{x}(k+1)=\left[\begin{array}{ll}
A & 0 \\
A & 0
\end{array}\right]\left[\begin{array}{l}
x(k) \\
\tilde{x}(k)
\end{array}\right]+\left[\begin{array}{cc}
B & 0 \\
B & I_{n_{x} * n_{x}}
\end{array}\right]\left[\begin{array}{l}
u(k) \\
\tilde{u}(k)
\end{array}\right] \\
\text { Enfoque UTE, V.9-N.1, Mar.2018, pp. } 204-216
\end{gathered}
$$


Además, los pesos de la función de coste $Q$ y $R$ se definen como:

$$
\hat{Q}=\left[\begin{array}{cc}
Q & 0 \\
A & \alpha Q
\end{array}\right], \quad R=\left[\begin{array}{cc}
R & 0 \\
A & \beta R
\end{array}\right]
$$

Donde, estos valores de $\alpha$ y $\beta$ son los pesos en la función de coste para los estados adicionales y la entrada de control respectivamente. Además, $\alpha$ debe ser un valor alto para penalizar la desviación de los estados de las referencias y $\beta$ debe ser un valor pequeño para dar libertad a la entrada de control en el momento de dirigir el sistema hacia sus referencias. El problema de optimización que incorpora restricciones suaves se establece de la siguiente manera

Sujeto a (30):

$$
\{\hat{u}[k], \ldots, \hat{u}[k+N-1]\} \sum_{i=k}^{k+N_{p}-1} J(\hat{u}[i+1], \hat{u}[k]
$$

$$
\begin{gathered}
\hat{x}[i+1] \in X, \forall i \in \mathbb{Z}_{k}^{k+N_{p^{-}}-1} \\
u[i] \in U, \forall i \in \mathbb{Z}_{k}^{k+n_{p}-1}
\end{gathered}
$$

Nótese que las entradas de control $\tilde{u}[k]$ no están sujetas a restricciones debido a estas acciones de control que pueden tomar valores en un amplio rango.

\section{Resultados y discusión}

En esta sección, los dos controladores son implementados mediante simulación para comparar su rendimiento y destacar sus ventajas y desventajas.

\subsection{Resultados del Controlador PI-LQR}

La acción integral en el LQR es proporcionada por constantes óptimas multiplicadas por el error. En este punto, la matriz de ganancia para el sistema de cuatro tanques está dada por:

$$
K_{a}=\left[\begin{array}{cccccccc}
-0.3819 & -1.1327 & 0.3513 & -2.1857 & -2.6174 & 9.6603 & -6.5885 & 12.4323 \\
-1.3516 & -0.6658 & -2.0236 & 0.2783 & 12.9532 & -1.3903 & 13.8071 & -5.5907
\end{array}\right]
$$

Las acciones de control se calculan según los pesos $R$ y $Q$ para dar más prioridad a los estados que a las entradas del sistema.

En este marco de simulación, la condición inicial es $h[0]=0.65 \mathrm{~m}$ para cada tanque que corresponde al punto de operación. Las referencias para cada estado se establecen en $0.5 \mathrm{~m}$ y tienen las mismas matrices $R$ y $Q$ cuyos valores se especifican de acuerdo con (26).

La Figura 3 muestra que el LQR ayuda a alcanzar la referencia en cada tanque, la respuesta contiene un error de estado estable que es mínimo.

Para producir estos resultados, los flujos de entrada requeridos $q_{A}$ y $q_{B}$ se muestran en la Figura 4. Como puede verse, la entrada B $\left(q_{B}\right)$ comienza antes de la entrada $\mathrm{A}\left(q_{A}\right)$ en $\mathrm{t}=0$ que proporcionan un flujo máximo de $1.4 \mathrm{~m}^{3} / \mathrm{h}$ y $1.8 \mathrm{~m}^{3} / \mathrm{h}$, respectivamente. Es importante notar que ambas señales de control no alcanzan el flujo de saturación de $3.26 \mathrm{~m}^{3} / \mathrm{hy} 4 \mathrm{~m}^{3} / \mathrm{h}$, dado por (5). 


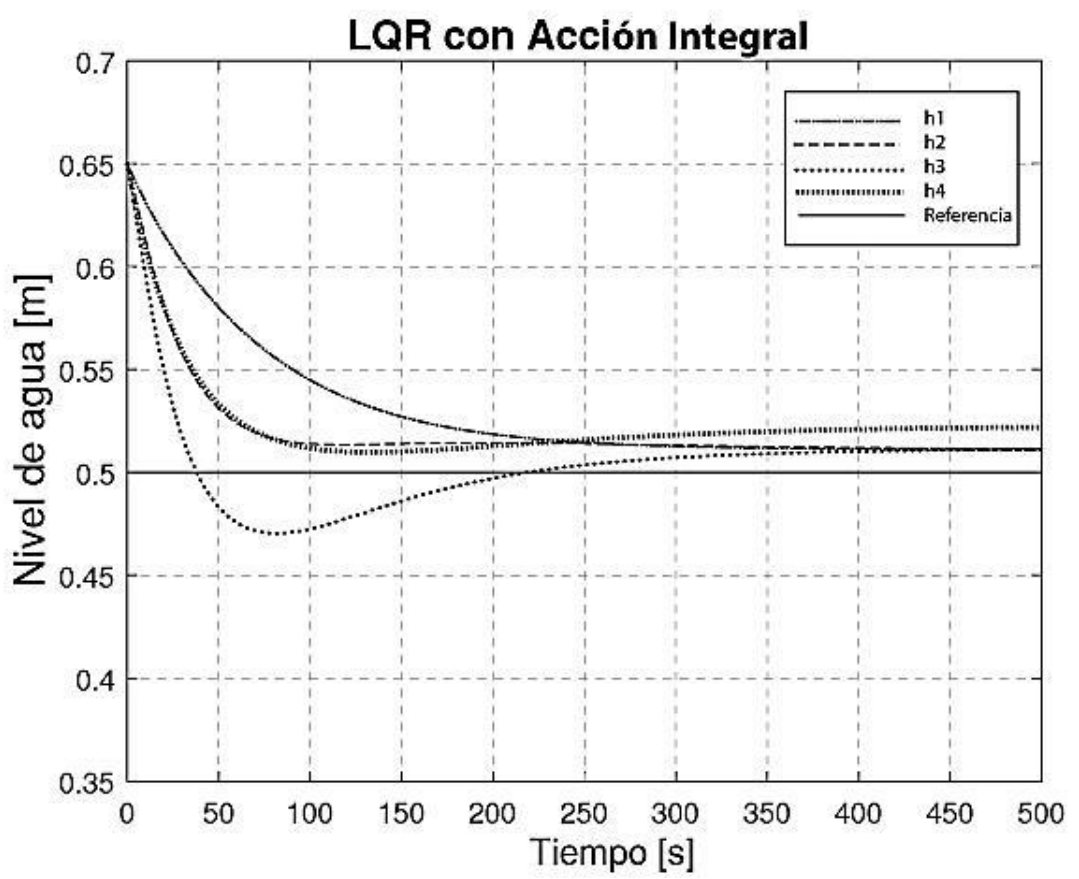

Figura3. Nivel de agua empleando LQR con acción integral

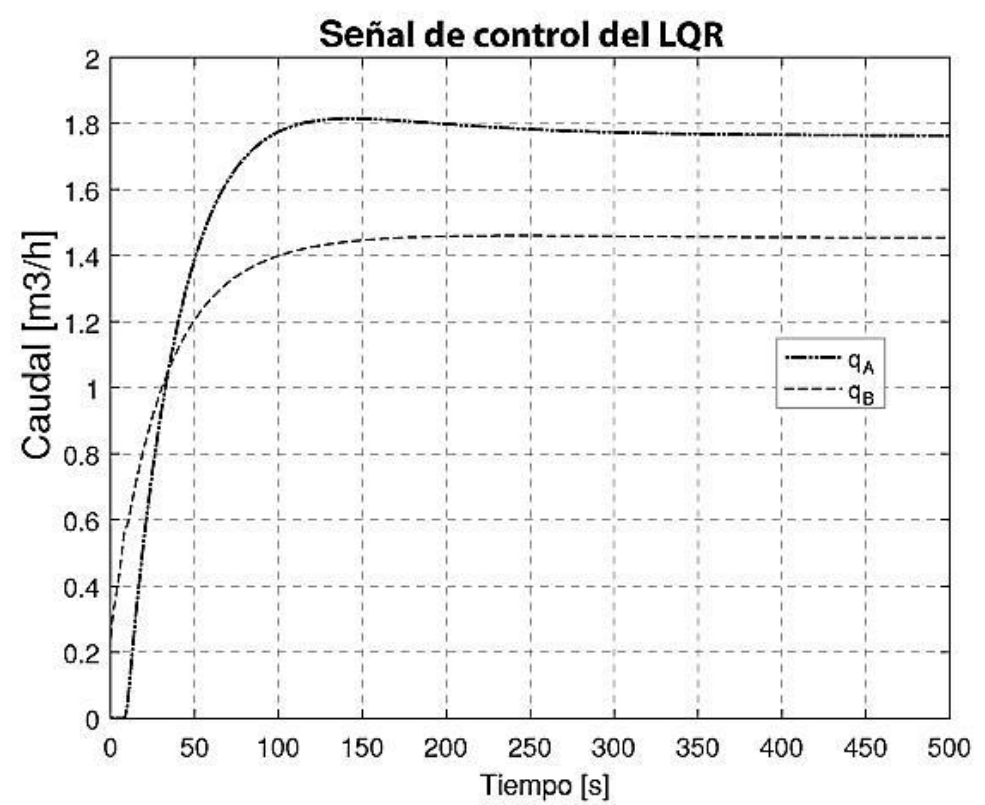

Figura 4. Acciones de control empleando PI-LQR

\subsection{Resultados del Controlador SC-MPC}

El error en estado estacionario con el SC-MPC se reduce por la acción de las restricciones suaves que producen un alcance rápido de las referencias con grandes sobre-impulsos, además, se tiene un error mínimo de estado estacionario. La Figura 5 muestra las respuestas de la planta obtenidas al aplicar un controlador MPC en las mismas condiciones establecidas con el controlador LQR y con un horizonte de predicción $N_{p}=10$.

Debido a las restricciones suaves, ambas entradas de control comienzan después de $\mathrm{t}=0$ con un retardo. Luego de eso, estas señales de control empiezan a aumentar rápidamente hasta alcanzar flujos constantes de $1.4 \mathrm{~m}^{3} / \mathrm{h}$ y $1.8 \mathrm{~m}^{3} / \mathrm{h}$, respectivamente, como se muestra en la Figura 6. 


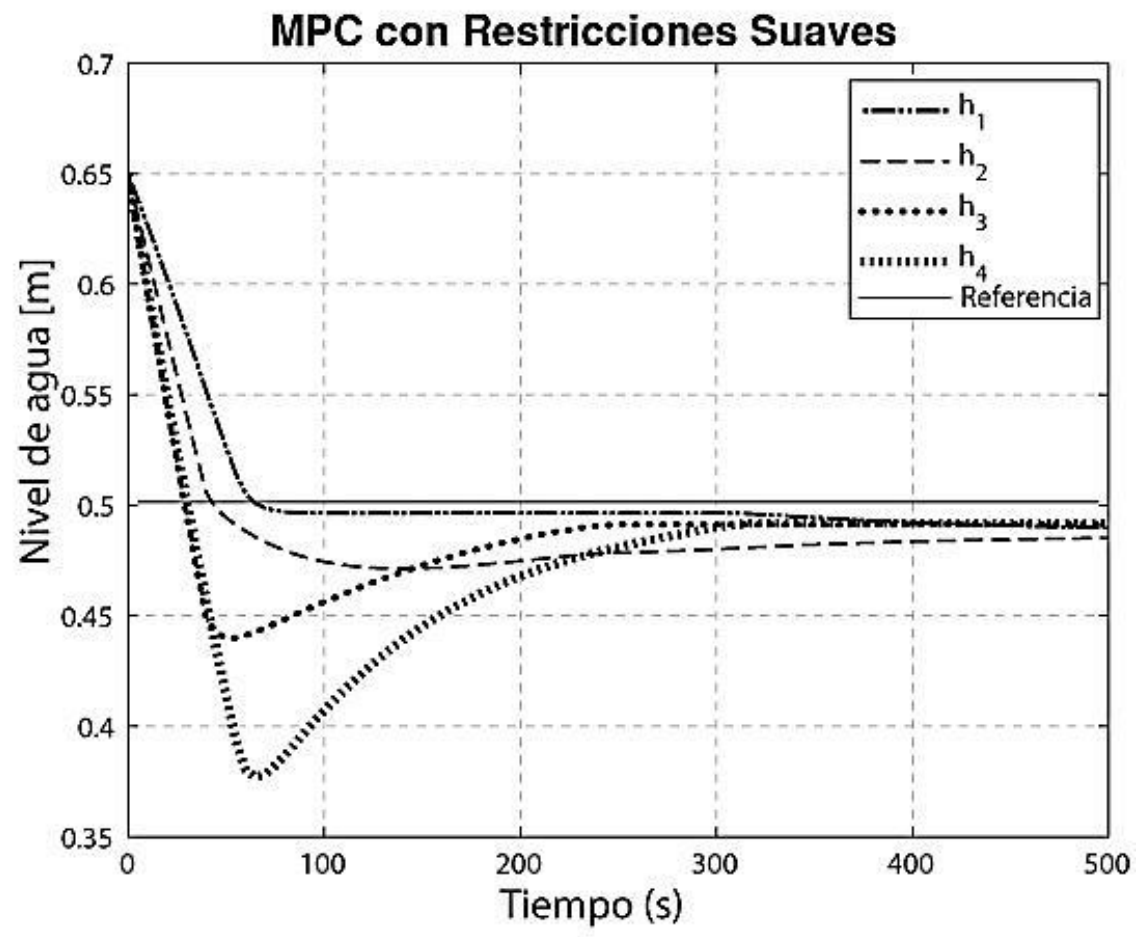

Figura 5. Nivel de agua empleando MPC con restricciones suaves

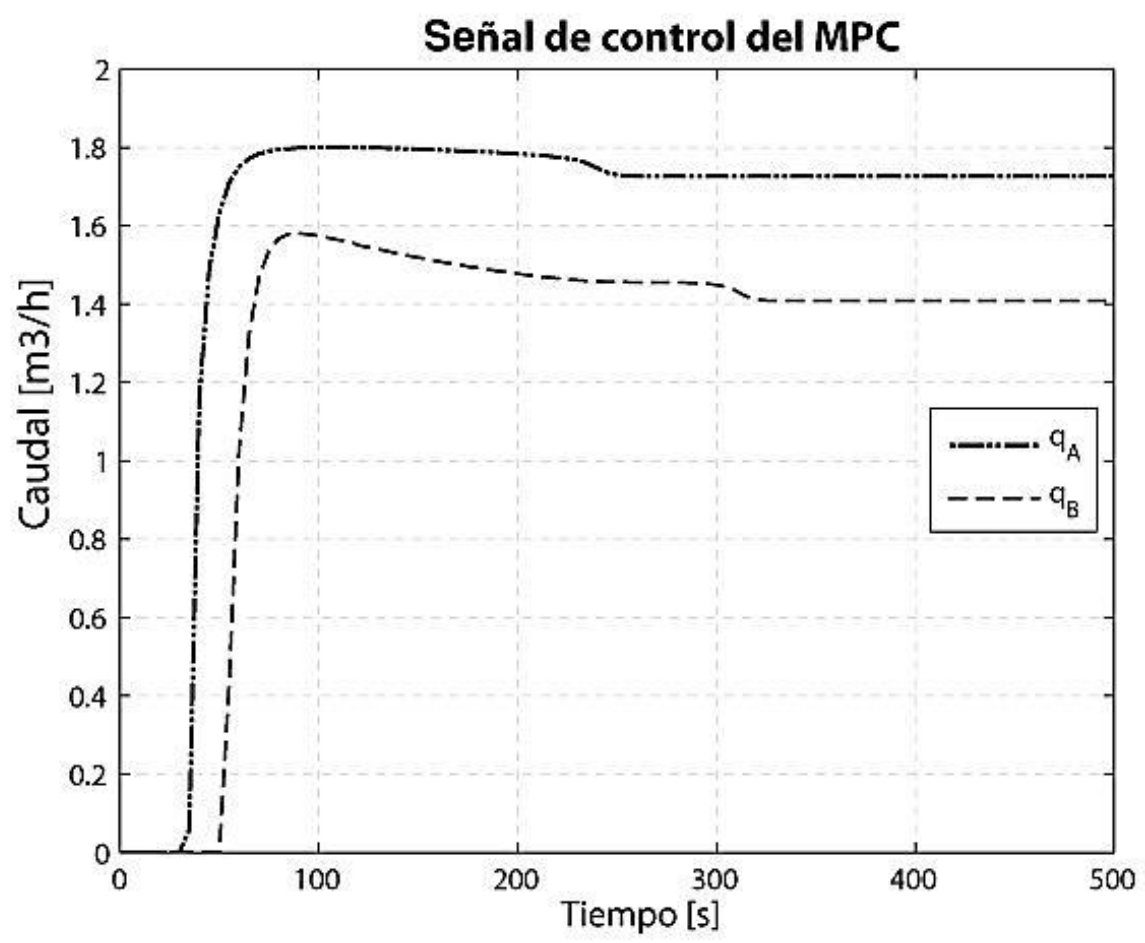

Figura 6. Acciones de control empleando SC-MPC

\subsection{Comparación de rendimiento}

El objetivo en la comparación entre los controladores SC-MPC y PI-LQR es determinar cuál produce la mejor respuesta mediante el análisis de los parámetros que muestren un índice de rendimiento como: el error de estado estable, el tiempo de ajuste y 
el tiempo de cálculo computacional. Ambos controladores funcionan en las mismas condiciones y tratan de reducir el error de estado estable a cero.

La capacidad del proceso para llegar a las referencias con entradas que hacen el menor esfuerzo para producir estas respuestas se denomina índice de rendimiento, este índice de rendimiento se calcula mediante la función de coste acumulada, dada por:

$$
\Phi_{\text {cost }}=\sum_{i=1}^{m_{x}}\left[\left(x[k]-x_{\text {ref }}\right)^{T} Q\left(x[k]-x_{\text {ref }}\right)+u^{T}[K] R * u[k]\right.
$$

Donde $m_{x}$ es el número de muestras tomadas durante el tiempo de simulación. Si se tiene en cuenta que el tiempo de muestreo seleccionado para el sistema de cuatro tanques es $\mathrm{T}=5 \mathrm{~s}$, esto resulta en:

$$
m_{x}=\frac{t_{s i m}}{T}
$$

Con un tiempo de simulación de $500 \mathrm{~s}$. El índice de rendimiento para los controladores son $P I-L Q R \phi_{\text {cost }}=50.106$ unidades de coste y $S C-M P C \phi_{\text {cost }}=47.1$ unidades de coste.

El rendimiento de salida de ambos controladores se puede comparar estableciendo un punto de referencia y midiendo los niveles de agua para cada uno de los tanques en estado estacionario y los tiempos de caída que se obtienen a partir de la Figura 3 y la Figura 5, estos resultados se resumen en la Tabla 2.

Tabla 2. Comparación de respuestas entre los controladores

\begin{tabular}{|l|l|l|l|l|}
\hline Estado Estable & h1 [m] & h2 [m] & h3 [m] & h4 [m] \\
\hline PI-LQR & 0.5129 & 0.5111 & 0.5149 & 0.5187 \\
\hline MPC & 0.5002 & 0.4878 & 0.5004 & 0.4996 \\
\hline Tiempo de bajada & $\mathbf{T}(\mathbf{h} 1)[\mathbf{s}]$ & $\mathbf{T}(\mathbf{h 2})[\mathbf{s}]$ & $\mathbf{T}(\mathbf{h} 3)[\mathbf{s}]$ & $\mathbf{T}(\mathbf{h 4})[\mathbf{s}]$ \\
\hline PI-LQR & 141.96 & 64.73 & 22.43 & 49.96 \\
\hline MPC & 51.05 & 37.65 & 28.90 & 28.90 \\
\hline Tiempo computacional & $\mathbf{T}[\mathbf{s}]$ & & & \\
\hline PI-LQR & $1.2 \times 10^{-7}$ & & & \\
\hline MPC & $6.04 \times 10^{-4}$ & & & \\
\hline
\end{tabular}

Los resultados indican que un controlador SC-MPC proporciona la respuesta más rápida con el menor error de seguimiento de referencia en estado estacionario y el coste acumulado final. Sin embargo, el tiempo de cálculo promedio requerido por el controlador SC-MPC es $6.04 \times 10^{-2} \mathrm{~s}$, este valor es considerablemente más grande que el PI-LQR que es en el orden de los microsegundos. Este parámetro, en ambos casos, no afecta el rendimiento del controlador debido a que el muestreo se realiza cada $5 \mathrm{~s}$ y es mucho mayor que el tiempo de cálculo para ambos controladores. El controlador SC-MPC agrega un retraso en la señal de control; debido al tiempo empleado en su cálculo; Por otro lado, la salida de control en el PI-LQR responde instantáneamente, sin embargo, el establecimiento es aproximadamente un 49\% más rápido con el MPC. Como se indicó anteriormente, el LQR tiene un pequeño error de posición el cual se mejoró alrededor del $2.24 \%$ con el controlador SC-MPC como se indica en la Tabla 2.

\section{Conclusiones y Recomendaciones}

Las respuestas de los controladores LQR y MPC en su forma común poseen un error de posición considerable. Una acción integral agregada al LQR y restricciones suaves al MPC permiten reducir este error considerablemente y mejorar los tiempos de respuesta. El sistema de cuatro tanques, que se modeló y linealizó en un espacio discreto, tiene un tiempo de muestreo relativamente grande de $5 \mathrm{~s}$. El cálculo de las acciones de control de ambos controladores toma menos que este tiempo de muestreo, sin embargo, la salida de control empleando SC-MPC requiere un esfuerzo 
computacional mayor que el PI-LQR lo que hace que el tiempo de cálculo con el LQR sea casi tres veces más rápido que con el SC-MPC.

Implementar un controlador LQR se vuelve más fácil porque solo requiere de una constante óptima calculada en forma off-line, mientras que un MPC requiere un cálculo en línea para estimar la salida de control en cada tiempo de muestreo, creciendo a medida que aumenta el horizonte de predicción.

El tiempo de ajuste se mejora con la implementación de restricciones suaves en el MPC lo cual significa que el sobreimpulso en cada estado también aumenta. Sin embargo, esta característica no afecta al rendimiento general del sistema

\section{Bibliografía}

Alvarado, I. y otros, 2011. A comparative analysis of distributed mpc techniques applied to the hd-mpc four-tank benchmark. Journal of Process Control, p. 800-8015.

Askari, M., Moghavvemi, M., Almurib, H. \& Muttaqi, K., 2016. Multivariable offset-free model predictive control for quadruple tanks system. Transactions on Industry Applications, p. 1882-1890.

Camacho, E. \& Bordons, C., 2004. Model Predictive Control in the Process Industry. Springer-Verlag.

Chamorro, W., Velarde, P. \& Mosquera, G., 2017. On the Performance Comparison between PI-LQR and Soft-Constrained MPC Applied to a 4-Tanks System. INCISCOS 2017.

Cheng, J. y otros, 2016. Robust finite-time sampled-data control of linear systems subject to random occurring delays and its application to four-tank system. Volumen 281:55-76.

Engules, D., Hot, M. \& B., A., 2015. Level control of a coupled-tank system via eigenvalue assignment and lqg control.. In Proceedings of the 23th Mediterranean Conference on Control and Automation (MED), p. 1198-1203.

Grosso, J. M. y otros, 2016. Stochastic model predictive control approaches applied to drinking water networks. Optimal Control Applications and Methods In Press,.

Guillod, O., Sarria, V., Kenfack, S. \& Pulgarin, C., 2003. An innovative coupled solarbiological system at field pilot scale for the treatment of biorecalcitrant pollutants. Issue 159(1):89-99.

H. Gouta, S. S. N. B. a. F. M., 2017. Generalized predictive control for a coupled four tank MIMO system using a continuous discrete time observer. ISA transactions, p. 280 292.

Herrera, M., Chamorro, W., Gomez, P. \& Camacho, O., 2015. Two-wheeled inverted pendulum robot nxt lego mindstorms Mathematical modelling and real robot comparisons. Revista Polit'ecnica.

Jimenez, A., Al-Hadithi, B. M., Alonso, L. \& Perez-Oria, J., 2013. Linear quadratic regulator based takagi-sugeno model for multivariable nonlinear processes. In System Theory, Control and Computing (ICSTCC), p. 285-290.

Johansson, K., 2000. The quadruple-tank process: a multivariable laboratory process with an adjustable zero. Volumen 8(3):456-465.

Mavrias, G., Fuchs, W. \& Binder, H. a. R. B., 2003. Anaerobic treatment of wastewater with high organic content using a stirred tank reactor coupled with a membrane filtration unit. Volumen 37(4):902-908.

Najarro, R., López, R., Racines, R. E. \& Puris, A., 2017. Un Algoritmo Genético Híbrido para la Optimización del Flow Shop Scheduling bajo Restricciones de Entornos Reales. Enfoque UTE, 8(5), pp. 14-25.

Sugeno, M. \& Takagi, T., 1985. Fuzzy identification of systems and its applications to modeling and control. IEEE Transactions on systems man, and cybernetics, p. 116132. 
Velarde, P., Valverde, L., Maestre, J. M. \& Ocampo-Martinez, C., 2017. On the comparison of stochastic model predictive contro strategies applied to a hydrogenbased microgrid. Journal of Power Sources, p. 161-173.

Vikram, K., Haizhou, P., Wong, M. \& de Queiroz, M., 2005. Experimental validation of a nonlinear backstepping liquid level controller for a state coupled two tank system. Issue 13(1):27-40,. 